\title{
A comparative randomized trial of intubation success in difficult intubation cases: the use of a Frova intubation catheter versus a Bonfils intubation fiberoscope
}

\author{
Ozkan Onal, Irem Gumus, Aysun Ozdemirkan, Faruk Cicekci, Mehmet Sarı, Hasan Huseyin Bayram, Cansu Ciftci, \\ Emine Aslanlar, Jale Bengi Celik \\ Department of Anesthesiology and Reanimation, Medical Faculty, Selcuk University, Konya, Turkey
}

Videosurgery Miniinv 2019; 14 (4): 486-494

DOI: https://doi.org/10.5114/wiitm.2019.83610

\begin{abstract}
Introduction: A difficult airway is one of the main causes of morbidity and mortality in patients who undergo surgical interventions. Therefore, many devices and algorithms have been developed for the management of a difficult airway. However no study has been conducted comparing Frova catheter (FC) and a Bonfils fiberoscope (BF) to date.

Aim: To compare the effectiveness and success of two devices, a FC and BF, in difficult intubation cases.

Material and methods: Design: Single-centre randomized controlled trial in patients with difficult airways. The assignment order was created by unplanned number charts, and the assignment was hidden in closed covers, which were not unlocked until case permission had been provided. Setting: The trial was undertaken in a university hospital in Turkey. The primary analysis was based on 60 participants $(n=30, n=30)$ with difficult intubation. The main outcomes were the success rates of placement of the tracheal tube in the trachea and the duration of the tracheal intubation process.

Results: In the BF group, successful intubation was carried out in 25 of the 30 (83.3\%) patients, whereas intubation was successful in 28 of the 30 patients (93.3\%) in the FC group. Patients who could not be intubated with the first device were intubated with the other device. The mean duration of intubation was 109 (85-140) s in the BF group, whereas it was $38.8(26-60) \mathrm{s}$ in the FC group.

Conclusions: Both devices were successful in difficult intubation cases. However, given the shorter duration of intubation using the $F C$ and its lower cost as compared with that of the $B F$, the $F C$ can be considered superior to the $B F$ in difficult intubation cases.
\end{abstract}

Key words: intubation, difficult airway, intratracheal, equipment and supplies, endoscopes, catheters, Frova, Bonfils.

\section{Introduction}

Many methods and devices have been developed to deal with anticipated or unexpected difficult airway problems and to decrease the difficulty of laryngeal imaging. These include videolaryngoscopes and fiberoptic bronchoscopes (FOBs), as well as the Fro- va catheter ( $\mathrm{FC}$ ) and Bonfils fiberoscope (BF). The BF (Karl Storz, Tuttlingen, Germany) is a hard, long, thin device, with a curved end. During intubation, a tracheal tube (TT) is placed in the shaft of the BF and inserted into the patient's mouth. It is then advanced to the opening of the glottis. After the vocal cords become visible, the TT is placed in the trachea $[1,2]$. 
Endotracheal intubation using the BF may be used for normal airways, but the BF is particularly useful in cases of difficult intubation, such as when a patient has limited cervical mobility or limited mouth opening $[3,4]$. The most important disadvantage of the BF is that it requires a long learning curve. Impaired visualization is an additional disadvantage of the BF and the main cause of BF intubation failure $[1,5]$. The latter is the result of the intraoral steamy appearance, secretions, or contamination. Difficult airway management guides recommend the early use of an intubating tube in cases of unexpected difficult intubations and always in cases of orotracheal intubations [6-9]. Intubating devices, such as bougies and stylets, make intubation easier in difficult airways. They are also relatively cheap, lightweight and easy to maneuver, and they require similar psychomotor ability to that acquired by a practitioner when learning tracheal intubation [10]. Thus, they are more likely to result in successful intubation when a patient presents with a restricted glottal opening [10]. The Frova Intubating Introducer (Cook U.K. Limited, Letchworth, Hertfordshire, U.K.) was introduced into clinical practice in 1998. The FC is a gum flexible bougie and offers improved endotracheal intubation. In addition to overcoming some limitations of videolaryngoscopes, the FC offers a range of benefits. For example, its distal tip bends anteriorly at $30^{\circ}$, thereby improving endotracheal biting of the instrument and allowing the instrument to be used with ease and without trauma [11]. Furthermore, the instrument is equipped with an adaptor, which means that the patient can be oxygenated and the endotracheal tube can be replaced if necessary. Finally, the FC overcomes restrictions that videolaryngoscopes today face in the manipulation and insertion of the endotracheal tube [12].

In the literature, many studies have used mannequins to compare devices used in cases of difficult intubation [13-16]. In studies carried out in the clinic, patients who are not candidates for difficult intubation are generally preferred when testing the performance of various intubation devices. There have only been a few studies on patients with difficult intubation [14, 17, 18]. To the best of our knowledge, no studies have compared the success of a videolaryngoscope and FC in cases of difficult intubation. Based on our clinical experience, we believe that the FC has a high rate of success in patients with difficult intubation and that the FC is superior to endoscopes and videolaryngoscopes in this respect.

\section{Aim}

In the present study, the success rates of FC and $B F$ were compared in patients with difficult intubation.

\section{Material and methods}

\section{Study design}

In a university hospital operating theatre, we randomized difficult intubation patients in a single blind, controlled clinical trial comparing the effectiveness and success of two devices.

The study was approved by Selcuk University Hospital's research ethics committee (Konya, Turkey), and written informed consent was obtained from all the patients prior to enrolment in the study.

Clinical trial registration: http://www.ANZCTR.org. au/ACTRN12619000058178.aspx.

\section{Study population}

We assessed 60 American Society of Anesthesiologists (ASA) physical status I-III adult patients aged 18-65 years who were scheduled to undergo elective surgical procedures requiring tracheal intubation and general anesthesia. All the patients were deemed as difficult intubation cases based on at least one unsuccessful intubation attempt by an anesthetist who had at least 3 years of experience. Difficult intubation included stylet use, changing the number of blades, and repositioning. Patients who were younger than 18 years and patients who were pregnant were excluded, as well as patients who had gastroesophageal reflux and delayed gastric emptying or severe pulmonary disease. In addition, emergency cases were excluded (Figure 1).

\section{Study intervention}

The study data were collected in the Selcuk University Medical Faculty operating theatres. The patients were randomized to two groups based on the intubation device used: tracheal intubation using a Macintosh laryngoscope assisted BF (a size 3 blade in females and a size 4 blade in males) (BF group) and tracheal intubation using a Macintosh laryngoscope assisted FC (a size 3 blade in females and a size 4 blade in males) (FC group). In cases when intubation could not be carried out with the chosen device at the third attempt by an anesthe- 


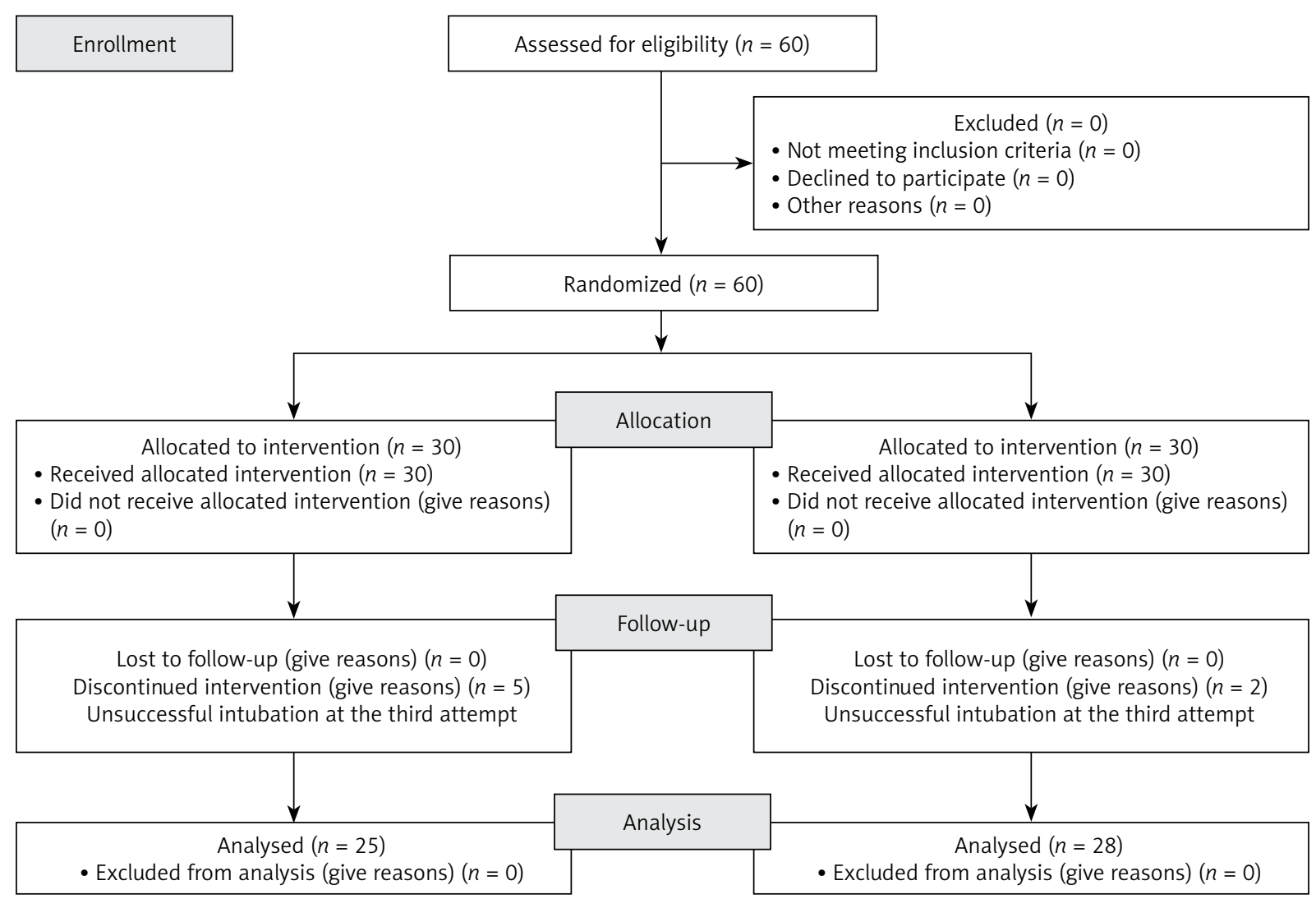

Figure 1. CONSORT 2010 flow diagram

tist with at least 3 years of experience, intubation was considered unsuccessful, and intubation was attempted with the second device. When this method was also unsuccessful, airway management was maintained according to established difficult airway algorithms [19]. In these cases, both devices were combined, or intubation was performed using an FOB. The duration of the first unsuccessful attempt, the Cormack-Lehane score (CLS) during the first attempt and with the chosen device, the success or not of the chosen device, the number of intubation attempts, the number of successful attempts, and the duration of successful attempts from the time of the placement of the blade intraorally to confirmation of tube insertion with capnography were recorded. In addition, the use of alternative methods, the type of alternative method preferred, the need for auxiliary maneuvers and stylets, whether a difficult mask was present, the occurrence of problems in extubation, the pressure exerted on the teeth (mild, moderate, or severe), and the lowest blood oxygen saturation $\left(\mathrm{SpO}_{2}\right)$ value recorded throughout the entire procedure were recorded. For all patients, the following data were recorded: the mandibular corner-mentum distance, thyromental distance, inter-incisor distance if the mouth opening was limited, and Mallampati score. In addition, data on neck movements (normal, limited, or absent); mandibular structure and tooth structure; the presence of a high larynx; a history of difficult intubation, head or neck surgery, or radiotherapy; systemic diseases that could lead to difficult intubation; traumas; and tracheostomies were recorded. Finally, obesity and the presence of a short neck were recorded.

All the patients received a general anesthetic. All the patients underwent electrocardiogram (ECG), noninvasive arterial pressure, $\mathrm{SpO}_{2}$ and end-tidal carbon dioxide monitoring $\left(\mathrm{ETCO}_{2}\right)$, as well as regular monitoring of volatile anesthetic grades. All the patients were preoxygenated via a numbered facemask and reached a fractional end-tidal oxygen $\left(\mathrm{ETO}_{2}\right)$ of at least 0.8 prior to the induction of anesthesia. General anesthesia was induced by fentanyl (Vem, Istanbul, Turkey) or remifentanil (Glaxo Smith Kline, Istanbul, Turkey) and propofol (Fresenius, Istanbul, Turkey) after neuromuscular blockade by 
rocuronium (Organon, Istanbul, Turkey). Following loss of consciousness, facemask ventilation was initiated, and anesthesia was provided with a volatile anesthetic factor (age-adjusted minimum alveolar concentration of 1.0). Two minutes after neuromuscular blockade, the laryngoscopy procedure was commenced by one of four anesthetists (I.G., O.O., J.B.C., or A.O.) practiced in the use of both devices ( $F C$ and $\mathrm{BF}$ ). Prior to this study, each of the anesthetists had performed more than 500 intubations using a Macintosh laryngoscope and at least 50 intubations using an FC or BF. Thus, the sufficiency of neuromuscular block in this 2-min period prior to intubation was not officially counted.

The single-use FC was guided into the trachea. The FC was then moved forward, and the user could sense "clicks" from the tracheal rings. The tracheal tube (TT) was then moved away above the FC to the trachea using the Seldinger procedure. The Macintosh laryngoscope and BF were placed inside the patient's mouth in a median line and pushed gently over the patient's tongue through the palatal wall up to the epiglottis. The blade was then pushed below the epiglottis, and the glottis was observed. A TT was then inserted into the shaft of the BF, inserted into the patient's mouth, and advanced into the opening of the glottis. After visualization of the vocal cords, the TT was inserted into the trachea. The trachea was intubated using a $7.5-8.5 \mathrm{~mm}$ TT in females and an 8.5-9.5 mm TT in males. Following tracheal intubation, the patient's lungs were mechanically ventilated for the period of the operation, and anesthesia was maintained with sevoflurane $(1.25-1.75 \%)$ in a mixture of nitrous oxide and oxygen in a $2: 1$ ratio. No other drugs or treatments were applied for $5 \mathrm{~min}$ after tracheal intubation. The administration of additional maintenance anesthesia was at the discretion of the anesthetist.

\section{Randomization}

The assignment order was created by unplanned number charts, and the assignment was hidden in closed covers, which were not unlocked until case permission had been provided.

\section{Study outcomes}

The main outcomes were the duration of the tracheal intubation process and the ratio of successful placement of the TT in the trachea. The intubation duration was considered the time from the biting of the blade by the teeth to the time the TT came into contact with the vocal cords, as determined by an anesthetist skilled in laryngoscopy. In cases where the TT was not straightly viewed, crossing the vocal cords straight, the intubation initiative was not considered complete until the TT bonded to the anesthetic circle and carbon dioxide was present in the expired breath. Unsuccessful intubation was considered failure to intubate the trachea or $>60 \mathrm{~s}$ to intubate the trachea. In these cases, care was taken to reduce any adverse effects of failed tracheal intubation. First, after unsuccessful tracheal intubation, the facemask was removed, and bag-mask ventilation was initiated. If the sufficiency of bag-mask ventilation could not be ascertained before or after intubation, the case was removed from the study, and intubation was performed according to the algorithm of the Difficult Airway Society for failed intubation [19]. Second, no more than three intubation efforts using the research instruments were allowed. However, in cases where the operator believed that there was little chance of success after the second failed attempt, no third attempt was undertaken, and the intubation device was deemed unsuccessful. In the event of unsuccessful intubation, a two-stage process was initiated. First, intubation attempts were permitted using the alternative device. If intubation using the alternative device also proved unsuccessful, the Difficult Airway Society failed intubation algorithm was continued [19]. The duration of the initial unsuccessful tracheal intubation procedure and that of the successful procedure were recorded.

Secondary outcomes included the number of intubation initiatives, the number of difficult mask ventilations, the number of optimization maneuvers needed (i.e., the use of a bougie and outer laryngeal manipulation with backward, upward, and rightward pressure), the need for an additional assistant to support tracheal intubation, and the CLS laryngoscopy score [20]. Additional secondary outcomes were arterial oxygen saturation levels before and shortly after the intubation attempt, minor injuries (e.g., lip or oral mucosa damage or blood on the laryngoscope), and spasms prior to extubation. All the data were collected by an independent observer. The assignment order was created by unplanned number charts, and the assignment was hidden in closed covers, which were not unlocked until case permission had been provided. 


\section{Sample size and statistical analysis}

We estimated that a total of 52 patients would be needed to detect a difference between groups, with a two-tailed $\alpha$ of 0.05 and a $(1-\beta)$ of 0.80 , for a comparison of 2 independent proportions in the composite outcome measure.

\section{Statistical analysis}

All data were evaluated using the software SPSS version 17.0. In the evaluation of the data, in addition to descriptive statistical methods (mean and standard deviation), cross tables were used. In the comparison of quantitative data, an independent two-sample $t$-test, Mann-Whitney $U$ test, and $\chi^{2}$ test

Table I. Sex distribution of successful intubation methods

\begin{tabular}{|c|c|c|c|}
\hline \multirow[t]{2}{*}{ Parameter } & \multicolumn{2}{|c|}{ Groups } & \multirow[t]{2}{*}{ Total } \\
\hline & $\mathrm{BF}$ & FC & \\
\hline \multicolumn{4}{|l|}{ Sex: } \\
\hline Male & 22 & 28 & 50 \\
\hline Female & 5 & 5 & 10 \\
\hline Total & 27 & 33 & 60 \\
\hline
\end{tabular}

Table II. Mean age and BMI in successful intubation methods

\begin{tabular}{|lccc|}
\hline Parameter & \multicolumn{2}{c}{ Groups } & P-value \\
\cline { 2 - 3 } & BF & FC & \\
\hline Age (mean \pm SD) & $48.77 \pm 12.16$ & $52.39 \pm 11.86$ & 0.250 \\
\hline BMI (mean \pm SD) & $27.87 \pm 5.35$ & $27.35 \pm 5.66$ & 0.717 \\
\hline BMI - body mass index. & & & \\
\hline
\end{tabular}

were used. All the findings were evaluated at the $95 \%$ confidence interval and $p<0.05$ significance level.

\section{Results}

A total of 60 cases were included in the research. Thirty cases were randomly selected to undertake tracheal intubation with either of the two instruments. Fifty of the $60(83.3 \%)$ patients in the present study were male. The male-female ratio was similar in the two groups. There were no significant between-group differences in terms of successful intubation, age, sex, and body mass index $(p>0.05)$ (Tables I, II).

The intubation success rate in the BF group was $83.3 \%$ (25/30) versus 93.3\% (28/30) in the FC group, but the difference was not statistically significant ( $p>0.05$ ) (Table III). In the BF group, 11 (36.7\%) patients were intubated at the first attempt, 11 (36.7\%) patients were intubated at the second attempt, and $3(10 \%)$ patients were intubated at the third attempt. As 5 (16.7\%) patients could not be intubated with the BF, intubation was attempted using the FC (Table III). In the FC group, 15 (50\%) patients were intubated at the first attempt, 12 (40\%) patients were intubated at the second attempt, and 1 (3.3\%) patient was intubated at the third attempt. As $2(6.7 \%)$ patients could not be intubated with the FC, they were intubated with the BF. The between-group comparison revealed no statistically significant difference in terms of the number of intubation attempts or the number of unsuccessful intubation attempts $(p>0.05)$ (Table III). In all cases, if a patient could not be intubated with the first method, intubation was successful with the second method. In total, 27 patients were intubated with the BF, and 33 patients were incubated with the FC (Table III).

Table III. Success rate of methods

\begin{tabular}{|c|c|c|c|c|}
\hline \multirow[t]{2}{*}{ Variable } & \multicolumn{2}{|c|}{ Groups } & \multirow[t]{2}{*}{ Total } & \multirow[t]{2}{*}{$P$-value } \\
\hline & $\mathrm{BF}$ & FC & & \\
\hline Successful intubation at the first attempt & $11(18.3 \%)$ & $15(25 \%)$ & $26(43.3 \%)$ & 0.40 \\
\hline Successful intubation at the second attempt & $11(18.3 \%)$ & $12(22.6 \%)$ & $23(43.3 \%)$ & \\
\hline Successful intubation at the third attempt & $3(5.6 \%)$ & $1(1.88 \%)$ & $4(6.6 \%)$ & \\
\hline Unsuccessful intubation & $\begin{array}{c}5(8.3 \%) \\
\text { (These } 5 \text { patients were } \\
\text { intubated with FC) }\end{array}$ & $\begin{array}{c}2(3.3 \%) \\
\text { (These } 2 \text { patients were } \\
\text { intubated with BF) }\end{array}$ & $7(11.6 \%)$ & \\
\hline Total successful intubation & $25(41.6 \%)$ & $28(46.6 \%)$ & $53(88.3 \%)$ & 0.424 \\
\hline
\end{tabular}


There was no statistically significant difference between the 27 patients intubated with the $\mathrm{BF}$ and the 33 patients intubated with the $F C$ with respect to the measured tiromental distance, mandibular mentum distance, and inter-incisor distance $(p>0.05)$ (Table IV).

The Mallampati score, which evaluates the airway and helps to predict difficult intubation, was not significantly different between the two groups $(p>0.05)$. However, there were more patients with a Mallampati score of 3 in the BF group and more patients with a Mallampati score of 4 in the FC group (Table V).

When the CLS was used to predict difficult intubation using the different intubation methods, there was a statistically significant difference $(p<0.05)$. More patients in the BF group had a CLS of 3, whereas more patients in the FC group had a CLS of 4 (Table V).

In the comparison of the duration of successful intubation cases using the two devices, the duration of intubation with the FC was significantly shorter than that of the BF $(p<0.05)$ (Table VI).

In terms of hemodynamic data, there was a significant between-group difference in the lowest $\mathrm{SpO}_{2}$ during intubation attempts $(p<0.05)$, with the lowest values found during intubation with the $\mathrm{BF}$ (Table VII).

\section{Discussion}

A difficult airway is a complex interaction of patient-related factors, the clinical environment, and the clinician's skills. Inadequate airway management may lead to hypoxic brain damage and death. The purpose of the current research was to compare the success rates of the $\mathrm{FC}$ and $\mathrm{BF}$ in difficult airway cases.

The BF was first used by Bonfils in 1983 for tracheal intubation of a child with Pierre Robin syndrome via a retromolar approach [21]. The BF can be employed in normal airways and difficult airways. It can also be used for awake intubation.

In a study by Kim et al. [22] that compared the success of intubation using an FOB and the BF in 40 patients with unexpected difficult laryngoscopy, successful intubation was faster using the BF than the FOB (77.9 and $145.5 \mathrm{~s}$, respectively). However, as intubation was not successful using the $B F$ in 2 patients, the success rates of the BF and FOB were
$80 \%$ and $100 \%$. respectively. In the same study, there was no statistically significant difference between the groups in terms of a sore throat, a hoarse voice, and hemodynamic changes during tracheal intubation. In the present study, the success rate of intubation was $83.3 \%(25 / 30)$ in the BF group. The

Table IV. Comparison of difficult intubation methods between successful intubation methods

\begin{tabular}{|lccc|}
\hline \multirow{2}{*}{ Parameter } & \multicolumn{2}{c}{ Groups } & P-value \\
\cline { 2 - 3 } & BF & FC & \\
\hline $\begin{array}{l}\text { Tiromental } \\
\text { distance } \\
\text { (mean } \pm \text { SD) }\end{array}$ & $7.16 \pm 1.25$ & $7.43 \pm 1.70$ & 0.402 \\
\hline $\begin{array}{l}\text { Inter-incisor } \\
\text { distance } \\
\text { (mean } \pm \text { SD) }\end{array}$ & $3.27 \pm 0.71$ & $3 \pm 0.99$ & 0.104 \\
\hline $\begin{array}{l}\text { Mandibular } \\
\text { mentum distance } \\
\text { (mean } \pm \text { SD) }\end{array}$ & $12 \pm 1.51$ & $12.33 \pm 1.30$ & 0.315 \\
\hline
\end{tabular}

Table V. Comparison of difficult intubation criteria between successful intubation methods

\begin{tabular}{|lccc|}
\hline \multirow{2}{*}{ Parameter } & \multicolumn{2}{c}{ Methods } & P-value \\
\cline { 2 - 3 } & BF & FC & \\
\cline { 1 - 3 } Mallampati 3 & $16(59.3 \%)$ & $12(21 \%)$ & 0.118 \\
\cline { 1 - 3 } Mallampati 4 & $11(40.7 \%)$ & $21(63.6 \%)$ & \\
\cline { 1 - 3 } CLS 3 & $16(59.3 \%)$ & $9(27.3 \%)$ & 0.018 \\
\cline { 1 - 3 } CLS 4 & $11(40.7 \%)$ & $24(72.7 \%)$ & \\
\hline
\end{tabular}

CLS - Cormack Lehane score.

Table VI. Comparison of duration of successful intubation between different methods

\begin{tabular}{|lccc|}
\hline Parameter & \multicolumn{2}{c}{ Groups } & P-value \\
\cline { 2 - 3 } & BF & FC & \\
\hline $\begin{array}{l}\text { Successful intuba- } \\
\text { tion duration [s] } \\
\text { (mean } \pm \text { SD) }\end{array}$ & $109 \pm 15.22$ & $38.8 \pm 10.89$ & $<0.001$ \\
\hline
\end{tabular}

Table VII. Comparison of hemodynamic values

\begin{tabular}{|lccc|}
\hline Parameter & \multicolumn{2}{c}{ Groups } & P-value \\
\cline { 2 - 3 } & $\mathrm{BF}$ & $\mathrm{FC}$ & \\
\hline $\begin{array}{l}\text { Minimum } \mathrm{SpO}_{2} \\
\text { value (mean } \pm \text { SD) }\end{array}$ & $94.70 \pm 1.29$ & $96.21 \pm 2.99$ & 0.033 \\
\hline
\end{tabular}


duration of intubation (109 s) in the present study was longer than that in the study by Kim et al. [22]. Our CLS scores of the patients explain the longer intubation duration. In their study, all the patients had a CLS of 3. In the present study, 14 of the 30 patients in the BF group had a CLS of 4 , whereas the other 16 patients had a CLS of 3, indicating that our patients had more difficult airways. In addition, there were no cases of sore throats, hoarse voices, or hemodynamic changes in the current study.

Byhahn et al. [23] simulated a difficult airway by restricting mouth opening in patients and having them wear a rigid cervical collar to restrict their neck movements. They then compared the success of intubations carried out with a BF and Macintosh blade. In the 76 patients included in their study, the rate of successful intubation using the Macintosh blade was $39.5 \%$ vs. $81.6 \%$ using the BF (two attempts). The duration of successful intubation was not different between the two groups (Macintosh blade: $53 \mathrm{~s}$; BF: 64 s). After removal of the cervical collar, all the patients were successfully intubated using the Macintosh laryngoscope. Byhahn et al. [23] demonstrated that the BF was a more effective intubation device for patients with immobilized cervical vertebrae and restricted mouth opening. They reported that the inability to advance the BF under the epiglottis due to the rigid nature of the device and the limited cervical movements and mouth opening were the main causes of the failure $(18.4 \%)$ in the BF group. In the present study, the duration of successful intubation was longer than that in the study by Byhahn et al. [23], and the rate of failure was $16.7 \%$. We think that the time is lower because it is performed only by cervical color insertion in patients without cervical immobilization. As in the aforementioned study, the latter was associated with the rigid nature of the BF, which meant it could not be advanced easily in patients with limited mouth opening. In addition, image quality was impaired by secretions.

Bein et al. [24] used a BF as a salvage device after unsuccessful intubation in 25 patients scheduled to undergo elective coronary bypass graft surgery. All the patients had difficult intubation after anesthesia induction and muscular relaxation. Intubation was first attempted using a Macintosh blade. All the patients had a CLS of 3 and 4 after two unsuccessful intubation attempts by an experienced anesthetist. Intubation was then attempted using the BF. Intubation with the BF was successful in 24 patients (a suc- cess rate of $96 \%)$. One attempt was unsuccessful due to oral secretions preventing visualization of the glottis. In this patient, tracheal intubation was performed successfully using an FOB. The mean duration of intubation using the BF was $47.5 \mathrm{~s}$. The present study also included some patients who could not be intubated using a Macintosh blade by an experienced anesthetist. These patients had CLSs of 3 and 4. The duration of successful intubation was longer in the present study, with lower success rates.

Rudolph et al. [25] compared intubation success rates using a $\mathrm{BF}$ and $\mathrm{FOB}$ in difficult intubation cases $(n=116)$ with CLSs of 3 or 4 . The mean duration of intubation was 169 s using the BF and 229 s using the FOB. There were three unsuccessful intubation attempts using the FOB, two of which were carried out successfully with the BF and one of which was carried out successfully with a Macintosh blade. The duration of successful intubation in their study was longer than that in the present study, but the success rate was higher in their study.

Following the introduction of the FC into clinical practice in 1998, a number of studies demonstrated that it had a high rate of success in difficult intubations [26-28]. The FC results in an anterior shift in the interarytenoid fold upwards toward the tracheal entrance. Due to its hollow structure, it can be connected to a respiratory circuit, and carbon dioxide can be measured [29]. Guides for difficult airway management recommend that in difficult intubation cases, endotracheal tube introducers should be used early and even routinely for orotracheal intubations [7-9].

In a study on 203 patients conducted in 2008, Hodzovic et al. [27] reported a success rate of $96 \%$ using an FC. The success rates were $84.2 \%, 12.3 \%$, and $1 \%$ at the first, second, and third attempts, respectively. Difficult intubation was not a criterion for inclusion in their study, and $17 \%, 57 \%, 26 \%$, and $1 \%$ of patients had CLSs of 1, 2, 3 and 4, respectively. In the present study, intubation was successful in $93.3 \%$ of patients using the $\mathrm{FC}$, and $50 \%$ of patients were intubated at the first attempt, $40 \%$ were intubated at the second attempt and 3.3\% were intubated at the third attempt. Among the patients, 27.35\% had a CLS of 3, and $72.75 \%$ had a CLS of 4 . The difference in the CLSs of the patients may explain the variance in the success rates of the two studies.

Janakiraman et al. [28] used a difficult intubation mannequin to compare the performance of an 
FC with new-Portex (Portex Tracheal Tube Introducer, Smiths Medical International, Hythe, UK) ProBreathe (Pro-Act Medical Ltd., Northampton, UK) and Eschmann (Smiths Medical International, Hythe, UK) tube changers and reported a high success rate of $78 \%$. In contrast, in a study by Hodzovic et al. [30] of mannequins with a CLS of 3 that were intubated using an FC with Portex and Eschmann introducers, the authors reported a success rate of $65 \%$. The anesthetists in these two studies had at least 1 year of experience. In contrast, the anesthetists in the present study had at least 3 years of experience. In addition, the patients in the present study had CLSs of 3 and 4 (i.e., difficult intubation cases), whereas the other two studies were conducted with mannequins having a CLS of 3 . The success rate in the present study was higher than that achieved in the studies by Janakiraman et al. [28] and Hodzovic et al. [30].

The limitations of our study are that more patients could have been included and pediatric patients were not included.

\section{Conclusions}

The $\mathrm{BF}$ and $\mathrm{FC}$ devices compared in the present study are strong, portable, and reliable, and they were both successfully used in difficult intubation cases. Although it takes longer to develop skills in the use of a BF as compared with the time taken to become skilled in the use of an FC, the BF is an efficient device in difficult intubations once expertise is gained. In the present study, the mean duration of intubation using the BF was longer than that using the FC. The longer duration was due to impaired vision due to secretions preventing visualization of the glottis and to difficulties in guiding the device due to its rigid construction. Moreover, the cost of the $\mathrm{BF}$ is significantly higher than that of the FC. Given the shorter duration of intubation and lower cost of the $F C$, we believe that the $F C$ is superior to the $B F$.

\section{Conflict of interest}

The authors declare no conflict of interest.

\section{References}

1. Lye ST, Liaw CM, Seet E, Koh KF. Comparison of results from novice and trained personnel using the Macintosh laryngoscope, Pentax AWS(R), C-MAC and Bonfils intubation fibrescope: a manikin study. Singapore Med J 2013; 54: 64-8.
2. Piepho T, Noppens RR, Heid F, et al. Rigid fiberscope Bonfils: use in simulated difficult airway by novices. Scand J Trauma Resusc Emerg Med 2009; 17: 33.

3. Rudolph C, Schneider JP, Wallenborn J, Schaffranietz L. Movement of the upper cervical spine during laryngoscopy: a comparison of the Bonfils intubation fibrescope and the Macintosh laryngoscope. Anaesthesia 2005; 60: 668-72.

4. Shollik NA, Ibrahim SM, Ismael A, et al. Use of the Bonfils intubation fiberscope in patients with limited mouth opening. Case Rep Anesthesiol 2012; 2012: 297306.

5. Halligan M, Charters P. A clinical evaluation of the Bonfils intubation fibrescope. Anaesthesia 2003; 58: 1087-91.

6. Sorbello M, Frova G. Frova introducer: Neither a stylet nor simply an introducer. Anaesthesia 2008; 63: 1010-3.

7. Practice guidelines for management of the difficult airway: an updated report by the American Society of Anesthesiologists Task Force on Management of the Difficult Airway. Anesthesiology 2003; 98: 1269-77.

8. Henderson JJ, Popat MT, Latto IP, Pearce AC; Difficult Airway Society. Difficult Airway Society guidelines for management of the unanticipated difficult intubation. Anaesthesia 2004; 59: 675-94.

9. Fischler M, Bourgain JL, Chastre J, et al. Difficult airway; teaching strategies and techniques: question 7. Socie'te' Franc, aise d'Anesthe'sie et de Re'animation. Ann Fr Anesth Reanim 2008; 27: $54-62$.

10. Messa MJ, Kupas DF, Dunham DL. Comparison of bougie-assisted intubation with traditional endotracheal intubation in a simulated difficult airway. Prehosp Emerg Care 2011; 15: 30-3.

11. Sharma R. A new maneuver for endotracheal tube insertion during difficult glidescope intubation: a suggestion. J Emerg Med 2011; 40: 443.

12. Xue FS, Cheng Y, Li RP, Liao X. Comparative performance of direct and indirect laryngoscopes for emergency intubation under cervical stabilization. Resuscitation 2012; 83: e169.

13. Niforopoulou P, Pantazopoulos I, Demestiha T, et al. Video-laryngoscopes in the adult airway management: a topical review of the literature. Acta Anaesthesiol Scand 2010; 54: 1050-61.

14. Ng I, Hill AL, Williams DL, et al. Randomized controlled trial comparing the McGrath videolaryngoscope with the C-MAC videolaryngoscope in intubating adult patients with potential difficult airways. Br J Anaesth 2012; 109: 439-43.

15. Wetsch WA, Spelten O, Hellmich M, et al. Comparison of different video laryngoscopes for emergency intubation in a standardized airway manikin with immobilized cervical spine by experienced anaesthetists. A randomized, controlled crossover trial. Resuscitation 2012; 83: 740-5.

16. Theiler L, Hermann K, Schoettker P, et al. SWIVIT - Swiss video-intubation trial evaluating video-laryngoscopes in a simulated difficult airway scenario: study protocol for a multicenter prospective randomized controlled trial in Switzerland. Trials 2013; 14: 94.

17. Malik MA, Subramaniam R, Maharaj $\mathrm{CH}$, et al. Randomized controlled trial of the Pentax AWS, Glidescope, and Macintosh laryngoscopes in predicted difficult intubation. $\mathrm{Br} J$ Anaesth 2009; 103: 761-8. 
18. Aziz MF, Abrons RO, Cattano D, et al. First-attempt intubation success of video laryngoscopy in patients with anticipated difficult direct laryngoscopy: a multicenter randomized controlled trial comparing the C-MAC D-BladeVersus the GlideScope in a mixed provider and diverse patient population. Anesth Analg 2016; 122: 740-50.

19. Apfelbaum JL, Hagberg CA, Caplan RA, et al.; American Society of Anesthesiologists Task Force on Management of the Difficult Airway. Practice Guidelines for Management of the Difficult Airway An Updated Report by the American Society of Anesthesiologists Task Force on Management of the Difficult Airway. Anesthesiology 2013; 118: 251-70.

20. Cormack RS, Lehane J. Difficult tracheal intubation in obstetrics. Anaesthesia 1984; 39: 1105-11.

21. Bonfils P. Difficult intubation in Pierre-Robin children, a new method: the retromolar route. Anaesthesist 1983; 32: 363-7.

22. Kim SH, Woo SJ, Kim JH. A comparison of Bonfils intubation fiberscopy and fiberoptic bronchoscopy in difficult airways assisted with direct laryngoscopy. Korean J Anesthesiol 2010; 58: 249-55.

23. Byhahn C, Nemetz S, Breitkreutz R, et al. Brief report: tracheal intubation using the Bonfils intubation fibrescope or direct laryngoscopy for patients with a simulated difficult airway. Can J Anaesth 2008; 55: 232-7.

24. Bein B, Yan M, Tonner PH, et al. Tracheal intubation using the Bonfils intubation fiberscope after failed direct laryngoscopy. Anaesthesia 2004; 59: 1207-9.

25. Rudolph C, Henn-Beilharz A, Gottschall R, et al. The unanticipated difficult intubation: rigid or flexible endoscope? Minerva Anestesiol 2007; 73: 567-74.

26. Takenaka I, Aoyama K, Iwagaki T, et al. Approach combining the airway scope and the bougie for minimizing movement of the cervical spine during endotracheal intubation. Anesthesiology 2009; 110: 1335-40.

27. Hodzovic I, Wilkes AR, Stacey M, Latto IP. Evaluation of clinical effectiveness of the Frova single-use tracheal tube introducer Anaesthesia 2008; 63: 189-94.

28. Janakiraman C, Hodzovic I, Reddy S, et al. Evaluation of tracheal tube introducers in simulated difficult intubation. Anaesthesia 2009; 64: 309-14.

29. Frova G. Comparison of tracheal introducers. Anaesthesia 2005; 60: 516-7.

30. Hodzovic I, Latto IP, Wilkes AR, et al. Evaluation of Frova, single use intubation introducer in a manikin. Comparison with Eschmann multiple use introducer and Portex single-use introducer. Anaesthesia 2004; 59: 811-6.

Received: 4.10.2018, accepted: 4.02.2019. 